See Article page 262 .

\section{Commentary: Don't stress for the unnecessary stress}

\author{
Bahaaldin Alsoufi, MD
}

Sef and colleagues ${ }^{1}$ describe robot-assisted 2-patch repair in a 30-year-old patient with sinus venosus atrial septal defect (ASD) and partial anomalous pulmonary venous connection (PAPVC) of the right upper and middle lobes to the superior vena cava. The procedure was performed through a 4-cm right thoracotomy incision at the level of the anterior axillary line, in addition to 3 extra port sites. Arterial and venous cannulation was established via the right femoral artery and vein and the left internal jugular vein. The crossclamp time was 141 minutes and the cardiopulmonary bypass time was 190 minutes. The patient had normal sinus rhythm and was discharged home on the fifth postoperative day; he was doing well at 6month follow-up.

Over the past 2 decades, minimally invasive surgery has transformed the landscape of a wide range of surgeries, most notably abdominal and thoracic surgeries. For example, the use of video-assisted thoracic surgery increased steadily to surpass the number of open procedures performed in the current era. In a study from the Society of Thoracic Surgeons General Thoracic Surgery Database, video-assisted thoracic surgery anatomic lung resection increased from $8 \%$ in 2003 to $43 \%$ in $2009 .{ }^{2}$ That number increased in an updated analysis between 2012 and 2014 to $62 \%$. $^{3}$ More recently, robot-assisted surgery was developed and offered additional advantages in minimally invasive surgery, such as more familiar hand movements, enhanced vision, tremor reduction, greater precision, and overall improved suturing and tying speed. In cardiac surgery, the

\footnotetext{
From the Department of Cardiovascular and Thoracic Surgery, University of Louisville School of Medicine, Norton Children's Hospital, Louisville, Ky.

Disclosures: The author reported no conflicts of interest.

The Journal policy requires editors and reviewers to disclose conflicts of interest and to decline handling or reviewing manuscripts for which they may have a conflict of interest. The editors and reviewers of this article have no conflicts of interest.

Received for publication Aug 25, 2020; revisions received Aug 25, 2020; accepted for publication Aug 28, 2020.

Address for reprints: Bahaaldin Alsoufi, MD, Department of Cardiovascular and Thoracic Surgery, University of Louisville School of Medicine, Norton Children's Hospital, 201 Abraham Flexner Way, Suite 1200, Louisville, KY 40202 (E-mail: balsoufi@hotmail.com).

JTCVS Techniques 2020;4:269-70

2666-2507

Copyright (C) 2020 The Authors. Published by Elsevier Inc. on behalf of The American Association for Thoracic Surgery. This is an open access article under the CC BY-NCND license (http://creativecommons.org/licenses/by-nc-nd/4.0/).

https://doi.org/10.1016/j.xjtc.2020.08.080
}

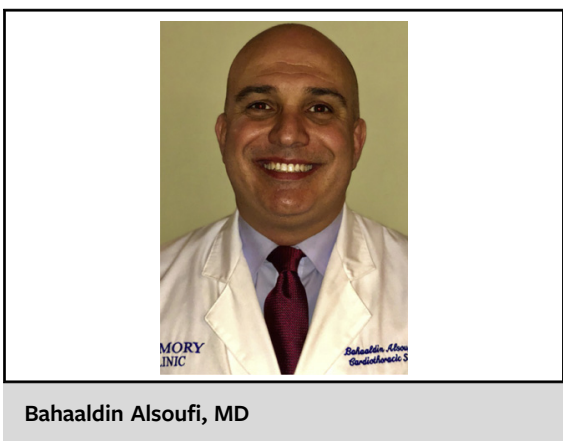

CENTRAL MESSAGE

Robotic sinus venosus ASD/

PAPVC repair in adults is doable

in experienced hands. Long

bypass and ischemic times, steep

learning curve, and unclear re-

covery advantage limit its bene-

fits to cosmetic only.

biggest utility for robot-assisted surgery has been with mitral valve repair and several centers have developed a great deal of experience with this technique and demonstrated superior results. ${ }^{4}$

The repair described in the case report by Sef and colleagues ${ }^{1}$ seems to have been performed by a cardiac surgeon who is likely very experienced with robot-assisted cardiac surgery on adults. However, the majority of patients with similar congenital anomalies are repaired during their childhood by cardiac surgeons who are usually not as skilled with robot-assisted surgery in pediatric populations. Therefore, when a new procedure is introduced, it seems prudent to examine its necessity, utility, and practicality. The following questions come to mind: What advantages does it offer over conventional open repair? Can it be performed as efficiently and safely as conventional repair? Can the results be replicated by the majority of surgeons? and, What is the cost-effectiveness of this procedure?

To answer these questions, one should look at the current expectations following repair of this relatively rare congenital anomaly, with focus on recovery and complications. In a large review of children with PAPVC from Toronto, a majority of patients had sinus venosus ASD and right-sided PAPVC. ${ }^{5}$ There were no early or late deaths, and the reintervention rate for vena cava or pulmonary venous obstruction was $<2 \%$. Although the incidence of sinus node dysfunction was not noted in that report, none of the patients 
received a permanent pacemaker. The average hospital stay was 3 days. Although the patients in that study were all children, it is safe to assume that the risk and recovery of an otherwise healthy 30-year-old patient described in the report by Sef and colleagues ${ }^{1}$ should be comparable. The robot-assisted procedure obviously avoids sternotomy, decreases wound length, and utilizes an axillary location with clear cosmetic results and less noticeable scar. This might translate into lower wound complication rate and potentially less post-operative bleeding, but it is not proven that this would result in better pain tolerance or earlier mobility. Additionally, the morbidity in cardiac surgery is not only related to sternotomy and pain, but also largely to the effect of cardiopulmonary bypass and cardiac ischemia on the heart and other organ function. In this case report, despite the fact that the surgeon who performed the surgery is experienced in robot-assisted surgery on adults, the bypass and cardiac ischemic times are at least double what is expected to be required when performing this repair with the traditional open technique. So, there is a trade off between the effects of sternotomy and cardiopulmonary bypass with this minimally invasive approach. Consequently, one should question if the potential benefits of this robot-assisted surgery justify exposing the heart to crossclamp time of 141 minutes compared with nearly 40 minutes and the patient to bypass time of 190 minutes compared with nearly 60 minutes. Additionally, one should question if these potential benefits justify the possible complications associated with percutaneous cannulation of the neck and femoral vessels. In the current report, the authors were able to perform the procedure safely and well; however, the recovery was not faster than expected, and although there were no complications related to cardiac rhythm or baffle obstruction, it is unlikely that the robot-assisted approach would lower these complications given that the same repair was essentially performed.

The experienced surgeons from West Virginia have elegantly and successfully repaired sinus venosus ASD and right PAPVC in an adult patient and that procedure might be a valid alternative strategy in the rare adult with similar presentation if performed by comparably experienced hands. Given the rarity of late presentation and management of this anomaly, the current deficient familiarity of the average congenital cardiac surgeons with robot-assisted surgery and the steep learning curve, I predict that this report will generate little enthusiasm to change surgical strategy, at least in the very near future. If you add to that the good early expectations and fast recovery with the current traditional surgery, the lack of clear advantage (other than cosmetic) in recovery or morbidity, the potential vascular morbidity, and the definite additional cost, my current reaction becomes: Why stress over the unnecessary?

\section{References}

1. Sef D, Wei LM, Rankin S, Spear CR, Gustafson RA, Badhwar V. Robotic-assisted two-patch repair of right partial anomalous pulmonary venous connection and sinus venosus defect. J Thorac Cardiovasc Surg Tech. 2020;4:262-4.

2. Ceppa DP, Kosinski AS, Berry MF, Tong BC, Harpole DH, Mitchell JD, et al. Thoracoscopic lobectomy has increasing benefit in patients with poor pulmonary function: a Society of Thoracic Surgeons database analysis. Ann Surg. 2012;256: 487-93.

3. Fernandez FG, Kosinski AS, Burfeind W, Park B, DeCamp MM, Seder C, et al. The Society of Thoracic Surgeons lung cancer resection risk model: higher quality data and superior outcomes. Ann Thorac Surg. 2016;102:370-7 [Erratum in Ann Thorac Surg. 2017;104:726.].

4. Mihaljevic T, Koprivanac M, Kelava M, Goodman A, Jarrett C, Williams SJ, et al. Value of robotically assisted surgery for mitral valve disease. JAMA Surg. 2014; 149:679-86.

5. Alsoufi B, Cai S, Van Arsdell GS, Williams WG, Caldarone CA, Coles JG. Outcomes after surgical treatment of children with partial anomalous pulmonary venous connection. Ann Thorac Surg. 2007;84:2020-6. 